\title{
Akzeleriert nicht unterlegen
}

Fragestellung: Ist eine akzelerierte Strahlentherapie bei metastatischer Rückenmarkkompression bei Patienten mit mäßiger bis schlechter Prognose einer konventionellen Fraktionierung gleichwertig?

Hintergrund: Bei Patienten mit metastatischer Rückenmarkkompression und drohendem Querschnitt müssen bei der Therapie prognostische Faktoren in Betracht gezogen und pragmatische Therapiemaßnahmen geprüft werden. Die Behandlung mit zehn Fraktionen zu 3 Gy ist ein typisches Vorgehen. Ziel der Therapie ist in erster Linie der Erhalt neurologischer Funktionen, während das Überleben in der Regel durch systemische Manifestationen der Grunderkrankung determiniert wird.

Patienten und Methodik: 203 Patienten mit metastatischer Rückenmarkkompression und mäßiger bis schlechter Prognose erhielten nach Ausschluss einer Operationsindikation randomisiert entweder $5 \times 4$ Gy in einer Woche oder 10 x 3 Gy in zwei Wochen. Der primäre Endpunkt war das Ansprechen nach einem Monat, definiert als Verbesserung oder Stabilisierung der motorischen Funktionen. Weitere Endpunkte waren lokale Tumorkontrolle und Gesamtüberleben nach einem, drei und sechs Monaten.

Ergebnisse: Nach einem Monat lagen die Ansprechraten bei 87,2\% im experimentellen und bei 89,6\% im Standardarm. Die Raten der Verbesserung und des Ausbleibens weiterer Progression sowie der Verschlechterung waren

Rades D, Segedin B, CondeMoreno AJ et al. Radiotherapy with 4 Gy $x 5$ versus 3 Gy $x 10$ for metastatic epidural spinal cord compression: Final results of the SCORE-2 Trial (ARO 2009/01). J Clin Oncol 2016; 34: 597-602

\section{Hier steht eine Anzeige.}

Springer

\section{- Kommentar von Michael Weller, Zürich, Schweiz}

\section{Das verkürzte Schema ist eine Alternative}

Die Autoren haben in einer schwierigen Indikation eine inhaltlich überzeugende randomisierte Studie durchgeführt, deren Ergebnisse nahelegen, dass bei entsprechender Patientenauswahl ein verkürztes Strahlentherapieschema von $5 \times 4$ Gy über eine Woche verabreicht werden kann. Bezüglich neurologischer Funktion waren beide Schemata vergleichbar, die Belastung der Patienten wurde auf eine Woche reduziert. Prognoseführend bezüglich des Überlebens bleibt die systemische Grunderkrankung. Zu beachten ist die Patientenauswahl, die aufgrund eines Scoring-Systems erfolgte: Die Studienergebnisse beziehen sich nicht auf Patienten mit günstigen prognostischen Faktoren oder Patienten, die für eine Operation geeignet sind.

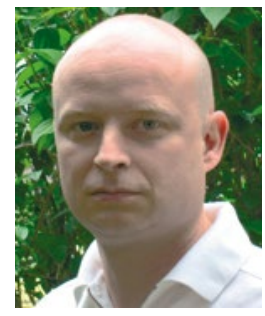

Prof. Dr. med. Michael Weller, Zürich/Schweiz

Direktor der Klinik für Neurologie,

Universitätsspital Zürich

E-Mail: michael.weller@usz.ch 